\title{
A fast current-driven instability in relativistic collisionless shocks
}

\author{
Martin Lemoine $^{1}{ }^{(a)}$, Guy Pelletier $^{2}{ }^{(b)}$, Laurent Gremillet $^{3}$ (c) and Illya Plotnikov² (d) \\ 1 Institut d'Astrophysique de Paris, CNRS, UPMC, 98 bis boulevard Arago, F-75014 Paris, France \\ 2 UJF-Grenoble 1 / CNRS-INSU, Institut de Planétologie et d'Astrophysique de Grenoble (IPAG) UMR 5274, F- \\ 38041 Grenoble, France \\ 3 CEA, DAM, DIF, F-91297 Arpajon, France
}

PACS 52.27.Ny - Relativistic plasmas

PACS 52.35.Qz - Microinstabilities

PACS 52.35.TC - Shock waves and discontinuities

\begin{abstract}
We report here on a fast current-driven instability at relativistic collisionless shocks, triggered by the perpendicular current carried by the supra-thermal particles as they gyrate around the background magnetic field in the shock precursor. We show that this instability grows faster than any other instability studied so far in this context, and we argue that it is likely to shape the physics of the shock and of particle acceleration in a broad parameter range.
\end{abstract}

Introduction. - Fast-growing electromagnetic micro-instabilities are central to various fields of physics, ranging from astrophysical collisionless shocks [1] to inertial confinement fusion and high-energy density plasmas 2]. Accordingly, such instabilities have received ample attention in recent years. Most notably, the celebrated counterstreaming Weibel/filamentation mode and its related beam-plasma instability variants (including the oblique two stream mode [3] ), have been thoroughly discussed in various scenarios, e.g. [4 9]. - Recent particle-in-cell (PIC) simulations 10 12 have demonstrated that this instability is the agent of mediation of unmagnetized collisionless shocks, through the build-up of an electromagnetic barrier on skin depth scales. Further simulations 1319 have shown that the self-generated turbulence provides the scattering centers required for the development of supra-thermal power-laws, as anticipated in [20]. Analytical studies have argued that this same instability may potentially account for the very efficient pre-heating of the electron population in weakly magnetized electron-ion shocks, up to near equipartition, which has important observational consequences in high energy astrophysics 21,22. Finally, the Weibel/filamentation instability may also potentially account for the large magnetization inferred in the

\footnotetext{
(a) E-mail: lemoine@iap.fr

(b) E-mail: guy pelletier@obs.ujf-grenoble.fr

(c) E-mail: laurent.gremillet@cea.fr

(d)E-mail: illya.plotnikov@obs.ujf-grenoble.fr
}

external blast of gamma-ray bursts [1].

In this context, the effective growth rate of the instability is a prime question, because the fastest mode tends to dominate other channels of instability (provided it is robust enough, of course). For relativistic shocks of finite magnetization, this issue becomes acute, because the rapid advection of the background plasma through the precursor prevents the growth of slow modes 8,23]. More specifically, at large Lorentz factors $\gamma_{\mathrm{sh}} \equiv\left(1-\beta_{\mathrm{sh}}^{2}\right)^{-1 / 2} \gg$ 1, and/or moderate magnetization levels $\sigma$ 1] even the Weibel/filamentation mode may not grow on a precursor crossing timescale. How the shock is structured in such conditions then remains an open question.

In the present Letter, we report on a current-driven instability which develops generically in a magnetized counterstreaming beam configuration. As we argue, this instability can grow faster than the Weibel/filamentation mode in the relativistic regime and it is the likely agent that shapes the collisionless shock in a large part of parameter space. Although we discuss this process in the framework of a relativistic collisionless pair shock, its range of applicability is potentially much broader, extending to the field of beam plasma physics.

The set-up that we have in mind is a beam of "suprathermal" particles gyrating in the magnetic field of the "background plasma" in the interpenetration region (e.g.,

\footnotetext{
${ }^{1} \sigma \equiv B_{\mid \mathcal{U}}^{2} /\left(4 \pi n_{\mid \mathcal{U}} m c^{2}\right)$, with $n_{\mid \mathcal{U}}$ the proper density of the background plasma, $B_{\mid \mathcal{U}}$ the magnetic field strength as measured in the background plasma rest frame $(\mathcal{U})$.
} 
the shock precursor); the gyrating particles produce an electrical current which is both transverse to the beam axis and to the background magnetic field; the compensation of this current by the background plasma then destabilizes a combination of the extraordinary mode and of the compressive modes of the plasma, with a growth rate possibly as large as $\omega_{\mathrm{p}}$ in the rest frame of the background plasma. In the following, we describe the development of the instability in the case of a relativistic pair shock; then, we discuss the relevance of this instability and argue that it can explain recent simulations of relativistic collisionless shocks.

\section{Current-driven instability. -}

Initial set-up. All throughout, we denote by $\mathcal{S}$ the frame in which the shock is at rest, $\mathcal{U}$ the frame in which the (far) upstream background plasma is at rest and, without loss of generality, we assume a shock with perpendicular magnetic field in the $\mathcal{S}$-frame, with normal incidence. In the $\mathcal{S}$-frame, outside the precursor, the background plasma flows in with 4 -velocity $u_{x, \infty \mid \mathcal{S}}=-\gamma_{\mathrm{sh}} \beta_{\mathrm{sh}}<0$ and proper density $n_{\mid \mathcal{U}}$. The precursor contains suprathermal $e^{+}-e^{-}$(shock-reflected or shock-accelerated), with mean Lorentz factor $\gamma_{\mathrm{sh}}$ and density $n_{\mathrm{b}}=\xi_{\mathrm{b}} \gamma_{\mathrm{sh}} n_{\mid \mathcal{U}}$, which rotate in the electromagnetic field carried by the background plasma, i.e., in a magnetic field $\boldsymbol{B}=B_{z} \boldsymbol{z}$ and convective electric field $\boldsymbol{E}=\beta_{\mathrm{sh}} B_{z, 0} \boldsymbol{y}$. Here, $\xi_{\mathrm{b}} \sim 0.1$ denotes the fraction of incoming shock energy transferred into the supra-thermal population; the numerical value is inferred from PIC simulations, e.g. [19]. To zeroth order, $B_{z}=B_{z, 0} \equiv \gamma_{\mathrm{sh}} B_{\mid \mathcal{U}}$ in terms of the background restframe field $B_{\mathcal{U}}$. The gyration of supra-thermal particles creates a current density $\boldsymbol{j}_{\mathrm{b}} \simeq-\xi_{\mathrm{b}} \gamma_{\mathrm{sh}} n_{\mid \mathcal{U}} e c \boldsymbol{y}$. The size of the precursor is set by the gyration radius of supra-thermal particles, since these particles execute a half-gyration orbit in the advected background electromagnetic field structure in the shock front rest frame $\mathcal{S}$; these particles have Lorentz factor $\gamma_{\mathrm{sh}}$ and gyrate in $B_{z} \simeq \gamma_{\mathrm{sh}} B_{\mid \mathcal{U}}$, therefore the size of this orbit is approximately $c / \omega_{\mathrm{c}}$, in terms of $\omega_{\mathrm{c}}=e B_{\mid \mathcal{U}} /(m c)$ the upstream cyclotron frequency.

In the $\mathcal{S}$ frame, the incoming background $e^{+} / e^{-}$drift along $\pm \boldsymbol{y}$ once they enter the precursor, with typical 3velocity $\beta_{y} \simeq \pm \xi_{\mathrm{b}}$, in order to compensate the current $\boldsymbol{j}_{\mathrm{b}}$. Their 4-velocity $y$-component reads: $u_{y} \simeq \pm \gamma_{\mathrm{sh}} \xi_{\mathrm{b}} c$, hence $\left|u_{y} / c\right| \gtrsim 1$ for relativistic shocks, quite possibly $\left|u_{y} / c\right| \gg 1$. As discussed in Ref. 8], current compensation is achieved to a very high degree. Therefore, in the following, we treat the supra-thermal particles as rigid and we study the stability of the system assuming full current compensation. We will discuss this assumption of a rigid current further below. Let us already point out that the plasma frequency of the supra-thermal particle beam is smaller than that of the background plasma, $\omega_{\mathrm{pb}} \simeq \xi_{\mathrm{b}}^{1 / 2} \omega_{\mathrm{p}}$ and that the growth rate of the currentdriven instability peaks at $\omega_{\mathrm{p}}$.

The deflection of the incoming flow along $\boldsymbol{y}$ implies a deceleration of the flow along $\boldsymbol{x}$ : the total $\mathcal{S}$-frame 3 -

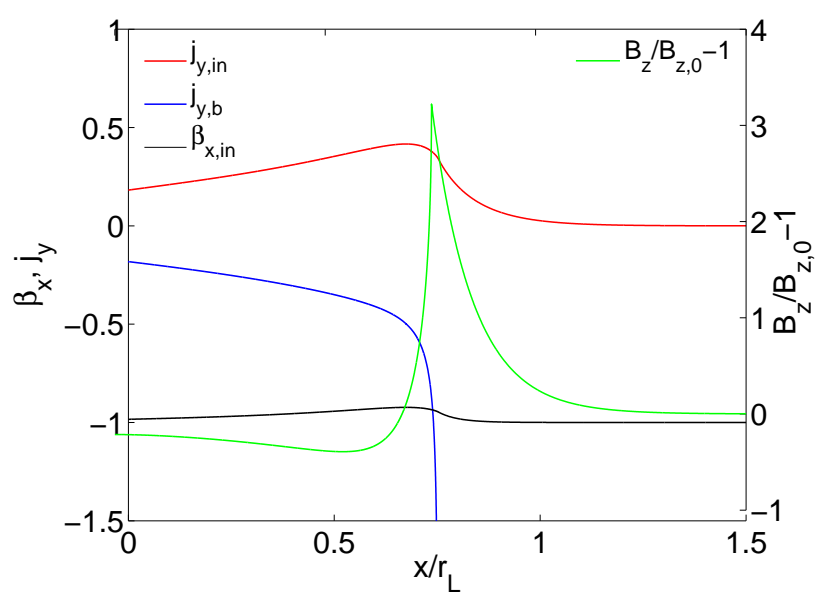

Fig. 1: Structure of the precursor as a function of distance to the shock, in the shock rest frame, for $\sigma=0.01, \xi_{\mathrm{b}}=0.1$ and $\gamma_{\mathrm{sh}}=1000$ : profiles of the normalized longitudinal velocity, $\beta_{x}$, transverse current density, $j_{y}$, and magnetic field perturbation, $B_{z} / B_{z, 0}-1$. The indices in and $\mathrm{b}$ refer to the background and supra-thermal populations.

velocity remains of order unity, $|\boldsymbol{\beta}| \sim 1$, up to corrections of order $\gamma_{\mathrm{sh}}^{-2}$ corresponding to the initial velocity; therefore, once $\left|\beta_{y}\right| \simeq \xi_{\mathrm{b}}, \beta_{x}$ has to deviate from unity by quantities of order $\gamma_{\mathrm{sh}}^{-2}$ or $\xi_{\mathrm{b}}^{2}$, whichever is larger. This leads us to define a new frame, $\mathcal{R}$, in which there is no bulk motion of the background plasma along $\boldsymbol{x}$. Assuming in particular $\gamma_{\mathrm{sh}} \xi_{\mathrm{b}} \gg 1$, as expected in ultra-relativistic shocks, one is led to $\left|\beta_{x}\right| \simeq 1-\xi_{\mathrm{b}}^{2} / 2$, meaning that the relative Lorentz factor between the shock front rest frame and the $\mathcal{R}$-frame has dropped from $\gamma_{\text {sh }}$ outside the precursor down to $\gamma_{\mathcal{R} \mid \text { sh }} \simeq 1 / \xi_{\text {b }}$ inside. This result holds in a more detailed calculation, which solves for the spatial profiles of the various fluids and of the electromagnetic field in a steady state cold fluid approximation; this model is described in a companion paper 24, but some salient features are illustrated in Fig. 11.

Note that the upstream plasma is only weakly decelerated as it enters the precursor, since the $x$-component $u_{x}$ of its 4 -velocity decreases by a relative amount $\simeq \xi_{\mathrm{b}}$. The total Lorentz factor of each electron and positron components of the background plasma is approximately conserved to order $\xi_{\mathrm{b}}$ as the components are simultaneously decelerated along $\boldsymbol{x}$ and accelerated along $\pm \boldsymbol{y}$.

Nevertheless, this deceleration along $\boldsymbol{x}$ has important consequences for the physics of the shock. In particular, the relevant frame in which to describe the instability as absolute (rather than convective) becomes $\mathcal{R}$; in this frame, the relative shock Lorentz factor with respect to the shock front is $\sim 1 / \xi_{\mathrm{b}}$ and the drift velocity along $y$ in $\mathcal{R}$ is ultra-relativistic, $\left|\beta_{y \mid \mathcal{R}}\right| \sim 1$, because of the Lorentz invariance of $u_{y}$.

Linear analysis. The relative drift of electrons and positrons in opposite directions in the $\mathcal{R}$ frame gives rise 


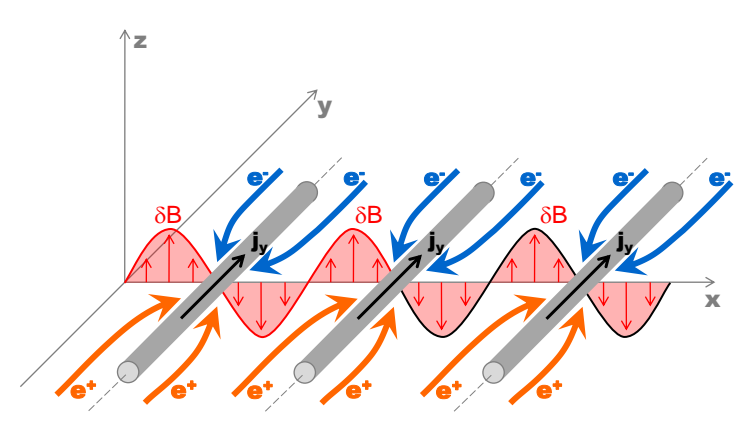

Fig. 2: Sketch of the development of the current-driven filamentation instability in the background plasma, with a perturbation along $\boldsymbol{x}$, in a 1D weakly magnetized limit. The external current carried by the supra-thermal particles is not shown here; see text for details.

to a current-driven instability, which can be intuitively understood in the weakly-magnetized two-dimensional limit $\sigma=B_{\mathrm{u}}^{2} /\left(4 \pi \gamma_{\mathrm{sh}}^{2} n_{\mid \mathcal{U}} m c^{2}\right) \ll \beta_{y}^{2}$ for transverse wavenumbers $\left(k_{y}=0\right)$. Then, it can be described as an analogue of the Weibel/filamentation instability, with a noticeable difference related to the opposite nature of the counterstreaming charges: in the former case, two counterstreaming beams of equal charges are deflected in opposite directions by a magnetic perturbation, thereby forming a charge perturbation which couples and feeds back positively onto the electromagnetic perturbation; this gives rise to filamentation with currents of opposite polarity in alternating filaments. In the present situation, the counterstreaming charges are opposite, thus they are deflected in the same direction at each point and they build a charge neutral density perturbation. This perturbation also sets up a perturbed current, which couples and feeds back positively onto the electromagnetic field. However, the instability now gives birth to filaments of equal polarity, oriented such as to compensate the external current induced by the supra-thermal particles (see Fig. 2). Such equalpolarity filaments are prone to coalescence, with potentially important consequences for the background plasma.

In order to illustrate the non-linear development of this current-driven instability in this weakly magnetized limit, we present in Fig. 3 a snapshot of 2D PIC simulations with the following set-up: a rigid external current $\mathbf{j}_{0}=-e n c / \sqrt{2} \boldsymbol{y}$ is imposed in the out-of-plane direction and the simulation box comprises a pair plasma at zero magnetization; the species drift at velocity $\beta_{y} \sim$ $1 /(2 \sqrt{2})$, leading to current-driven filamentation with a growth rate $\Im \omega \sim 0.2 \omega_{\mathrm{p}}$, in good agreement with expectations (see thereafter). The filaments coalesce rapidly: within $100 \omega_{\mathrm{p}}^{-1}$, there is only one remaining filament in the $43 c / \omega_{\mathrm{p}} \times 43 c / \omega_{\mathrm{p}}$ box. The magnetic field grows exponentially in the linear phase, then as a power-law in the non-linear phase, starting at $\sim 18 \omega_{\mathrm{p}}^{-1}$ (indicated by the
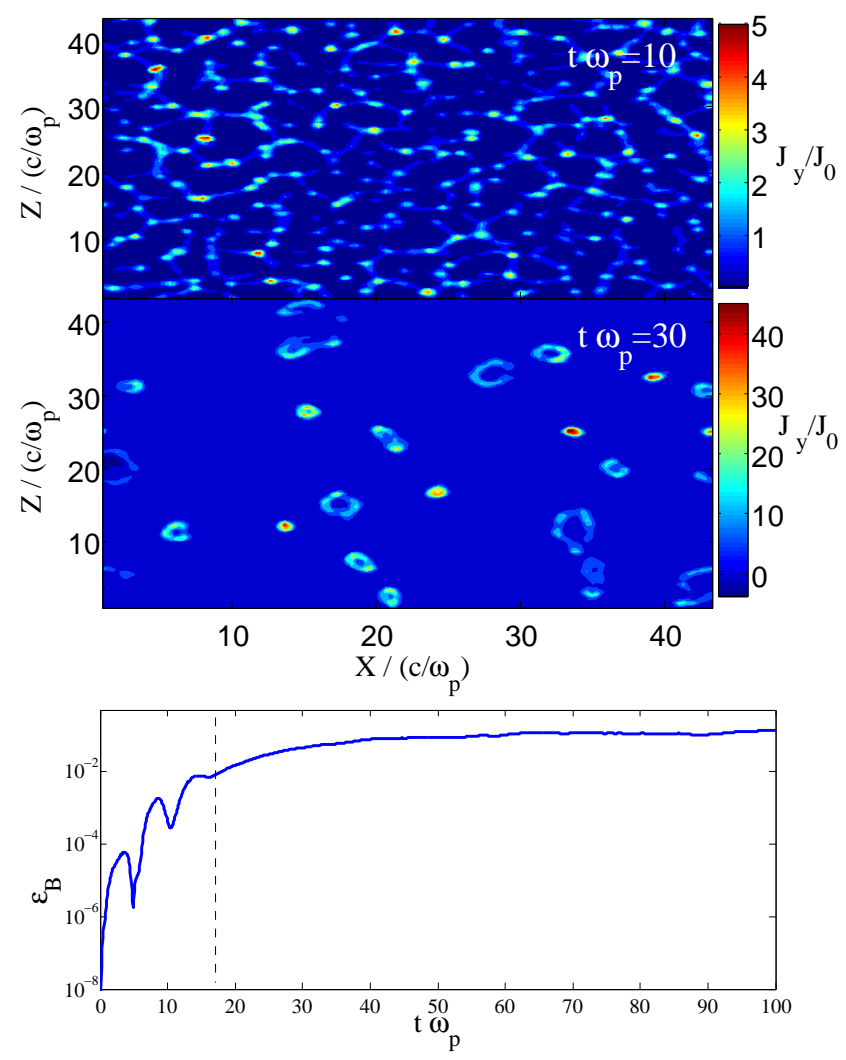

Fig. 3: 2D PIC simulation of the development of the currentdriven filamentation and of the coalescence phase. See text for details.

dashed line), up to a sub-equipartition value $\epsilon_{B} \sim 0.1$. We stress that such simulations do not aim at reproducing the development of this current-driven instability in the precursor of a relativistic shock, since the external current is here held rigid. However, they validate the general picture that we sketch here. Further simulations in more general configurations will be reported elsewhere.

In the previous unmagnetized cold plasma limit, one can derive directly the growth rate from the linear calculation of the Weibel/filamentation mode: the counter-streaming being symmetric, one expects an $\mathcal{R}$-frame growth rate $\Im \omega \simeq\left|\beta_{y \mid \mathcal{R}}\right| \omega_{\mathrm{p} \mid \mathcal{R}}$, with $\omega_{\mathrm{p} \mid \mathcal{R}}$ the (relativistic) plasma frequency of the electrons/positrons in the $\mathcal{R}$ frame. Here, $\left|\beta_{y \mid \mathcal{R}}\right| \sim 1$ and $\omega_{\mathrm{p} \mid \mathcal{R}} \simeq \omega_{\mathrm{p}}$, since the particles have for apparent density $\sim \gamma_{\mathrm{sh}} \xi_{\mathrm{b}} n_{\mid \mathcal{U}}$ in the $\mathcal{R}$ frame and a transverse motion of bulk Lorentz factor $\sim \gamma_{\mathrm{sh}} \xi_{\mathrm{b}}$. As a result, $\Im \omega \sim$ $\omega_{\mathrm{p}}$. To achieve such a fast growth rate in a highly asymmetrical beam configuration is quite remarkable. This property directly results from the fact that the external current imposes the counterstreaming within the background plasma itself, contrary to the Weibel/filamentation mode which results from the counterstreaming between the beam and the background plasma.

One can include finite magnetization effects in a twofluid model of the instability: for simplicity, we assume 
here $\beta_{y \mid \mathcal{R}}=\gamma_{\mathrm{sh}} \xi_{\mathrm{b}} \ll 1, k_{y}=0$ and a cold background plasma; $\beta_{y \mid \mathcal{R}} \ll 1$ means that we can omit the convective electric field in $\mathcal{R}$. In Fig. 4 , we provide a numerical solution to the full dispersion relation of the relativistic two-fluid model described in 24], for $\beta_{y \mid \mathcal{R}}=0.99$, in the 2D plane $\left(k_{x}, k_{y}\right)$, for $k_{z}=0$. This solution indicates that maximum growth then occurs at values $k_{y} \ll k_{x}$ (similarly, $k_{y} \ll k_{z}$ if $k_{x}=0$ ). With the present assumptions, one can look for a solution in which there is no charge density perturbation and $\Delta u_{x}=\Delta u_{z}=0$, with $\Delta u_{i} \equiv\left(\delta u_{i+}-\delta u_{i-}\right) / 2$ in terms of the respective velocity perturbations of the $+/-$ species. The instability involves velocity fluctuations $\delta u_{x}, \delta u_{z}-$ with the definition $\delta u_{i} \equiv\left(\delta u_{i+}+\delta u_{i-}\right) / 2-$ a density fluctuation $\delta n_{\mathrm{u}} \equiv\left(\delta n_{+,, \mathcal{U}}+\delta n_{-,, \mathcal{U}}\right) / 2$ and an electromagnetic perturbation characterized by the four-potential component $\delta A_{y}$. Then Maxwell's equations imply in Fourier variables

$$
\left(\omega^{2}-k^{2} c^{2}\right) \delta A_{y}=-4 \pi c \delta j_{y},
$$

with

$$
\delta j_{y}=2 e c n_{\mid \mathcal{U}}\left(\Delta u_{y}+\beta_{y \mid \mathcal{R}} \frac{\delta n_{\mid \mathcal{U}}}{n_{\mid \mathcal{U}}}\right) .
$$

From the equations of continuity for each species, one derives

$$
\frac{\delta n_{\mid \mathcal{U}}}{n_{\mid \mathcal{U}}}=\frac{k_{x} c}{\omega} \delta u_{x}+\frac{k_{z} c}{\omega} \delta u_{z}
$$

so that

$$
\left(k^{2} c^{2}-\omega^{2}\right) \delta \hat{A}_{y}=\frac{\omega_{\mathrm{p}}^{2}}{\omega_{\mathrm{c}}}\left(\Delta u_{y}+\beta_{y \mid \mathcal{R}} \frac{k_{x} c}{\omega} \delta u_{x}+\frac{k_{z} c}{\omega} \delta u_{z}\right) \text {. }
$$

The perturbations $\delta u_{x}, \delta u_{z}$ and $\Delta u_{y}$ are expressed in terms of $\delta \hat{A}_{y} \equiv \delta A_{y} / B_{\mathcal{U}}$ through the equations of motion. One thus ends up with the dispersion relation

$$
\left(P_{X}-\beta_{y \mid \mathcal{R}}^{2} \omega_{\mathrm{p}}^{2} k^{2} c^{2}\right) \omega^{2}+\beta_{y \mid \mathcal{R}}^{2} \omega_{\mathrm{p}}^{2} \omega_{\mathrm{c}}^{2} k_{z}^{2} c^{2}=0,
$$

where $k^{2} \equiv k_{x}^{2}+k_{z}^{2} . \quad P_{X}=0$ gives the dispersion relation of the extraordinary mode: $P_{X} \equiv \omega^{4}-$ $\left(\omega_{\mathrm{p}}^{2}+\omega_{\mathrm{c}}^{2}+k^{2} c^{2}\right) \omega^{2}+\omega_{\mathrm{c}}^{2} k^{2} c^{2}$.

The above derivation can be generalized by accounting for thermal effects through the inclusion of a finite sound velocity. Then the l.h.s. of Eq. (5) would be supplemented with a term which mixes the extraordinary and compressive modes 24]. Such finite temperature effects would quench the instability if $c_{\mathrm{s}} \gg \beta_{y \mid \mathcal{R}}$; however, one expects $\beta_{y \mid \mathcal{R}}$ close to unity at large $\gamma_{\text {sh }}$ while pre-heating effects in the precursor of pair shocks remain moderate 13]. The above Eq. (5) can be solved in the limit $\sigma \ll 1$ (but not necessarily $\sigma \ll \beta_{y \mid \mathcal{R}}^{2}$ ), leading to a negative root squared:

$$
\omega^{2} \simeq-\left(\beta_{y \mid \mathcal{R}}^{2}-\sigma\right) \omega_{\mathrm{p}}^{2}
$$

for $k c / \omega_{\mathrm{p}} \lesssim 1$. The growth rate thus becomes as high as $\omega_{\mathrm{p}}$ as $\beta_{y \mid \mathcal{R}} \rightarrow 1$; this scaling is confirmed by a multidimensional study of the instability, properly accounting for relativistic effects, see Fig. 4 and 24 .

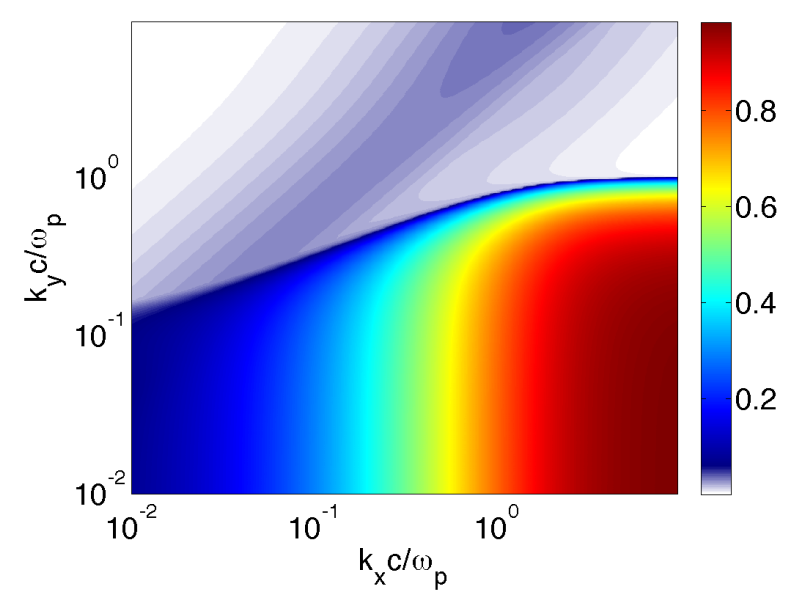

Fig. 4: Numerical calculation of $\Im \omega / \omega_{\mathrm{p}}$ for the full dispersion relation, including relativistic effects, assuming $k_{z}=0$, $\beta_{y \mid \mathcal{R}}=0.99, \sigma=10^{-3}$.

Discussion. - The above current-driven instability grows faster than any of the other instabilities discussed in the context of relativistic shocks. In particular, the transverse Weibel/filamentation instability growth rate is at most $\Im \omega \simeq \omega_{\mathrm{pb}}=\xi_{\mathrm{b}}^{1 / 2} \omega_{\mathrm{p}}(\mathcal{R}$-frame). One thus expects that the current-driven instability represents a key instability in collisionless shock physics and indeed, this can be argued as follows.

At relativistic collisionless shock waves of finite magnetization, the restricted timescale on which a plasma element crosses the precursor, $t_{\times \mid \mathcal{U}} \simeq\left(\gamma_{\mathrm{sh}} \omega_{\mathrm{c}}\right)^{-1}$ (written here in the background plasma rest frame) strongly constrains which instability can or cannot grow 8, 23]: in particular, neglecting any deceleration of the flow, the Weibel/filamentation instability cannot grow in the precursor if $\sigma \gtrsim \gamma_{\mathrm{sh}}^{-2} \xi_{\mathrm{b}}$ - a very low value if $\gamma_{\mathrm{sh}} \gg 1$ - as the growth timescale then becomes larger than $t_{\times \mid \mathcal{U}}$. In contrast, the present current-driven filamentation can grow in a much broader range of parameter space, due to the deceleration imparted to the background plasma: at large values of the shock Lorentz factor, i.e. $\gamma_{\mathrm{sh}} \xi_{\mathrm{b}} \gg 1$, the relevant frame in which to discuss the instabilities as absolute has become $\mathcal{R}$, which moves with a $\gamma_{\mathrm{sh}}$-independent Lorentz factor $\gamma_{\mathcal{R} \mid \text { sh }} \sim 1 / \xi_{\mathrm{b}}$ relatively to the shock front. In this $\mathcal{R}$-frame, the precursor crossing timescale becomes $t_{\times \mid \mathcal{R}} \simeq 1 /\left(\gamma_{\mathcal{R} \mid \mathrm{sh}} \omega_{\mathrm{c}}\right)$; hence, the current-driven instability can grow whenever $\Im \omega t_{\times \mid \mathcal{R}} \gtrsim 1$, or

$$
\sigma \lesssim \xi_{\mathrm{b}}^{2}
$$

For $\sigma \lesssim 10^{-2}$ (assuming $\xi_{\mathrm{b}} \sim 0.1$ ), this implies that the current-driven filamentation can grow at any value of the shock Lorentz factor. Therefore, at large Lorentz factors and/or moderate magnetization levels, in particular $\gamma_{\mathrm{sh}}^{2} \sigma \xi_{\mathrm{b}}^{-1} \gtrsim 1$ such that the Weibel/filamentation instability cannot grow, this current-driven instability is expected to shape the physics of the precursor. 


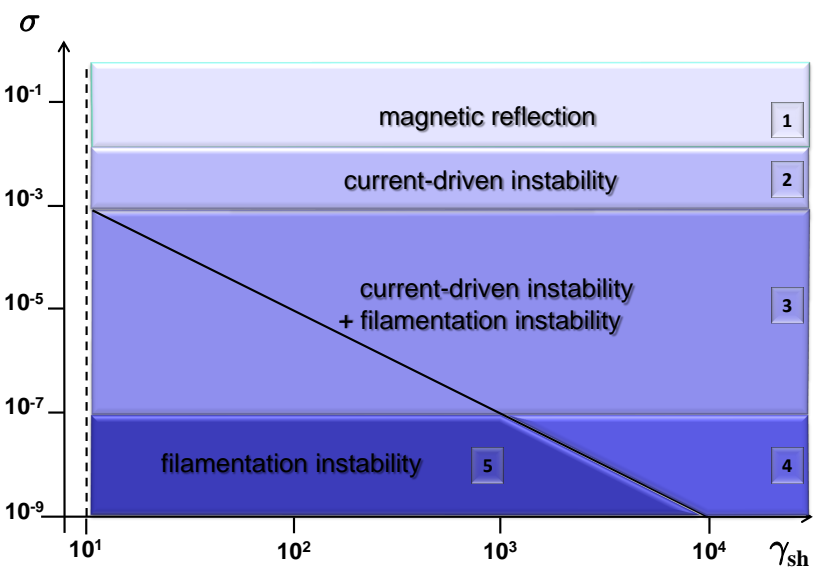

Fig. 5: Phase diagram of relativistic $e^{+} e^{-}$collisionless shocks in the plane $\left(\gamma_{\mathrm{sh}}, \sigma\right)$. Our calculations apply to the highly relativistic regime, to the right of the dashed line $\left(\gamma_{\mathrm{sh}} \gtrsim 10\right)$; this figure assumes $\xi_{\mathrm{b}}=0.1$. The various regions indicate the zones of influence of various instabilities and processes. Region 1: the shock transition is initiated by magnetic reflection; region 2 : the current-driven instability dominates, for $\sigma \lesssim \xi_{\mathrm{b}}^{2}=10^{-2}$ region 3: for $\sigma \lesssim \xi_{\mathrm{b}}^{3}=10^{-3}$, both the current-driven and the filamentation instability can grow, thanks to the deceleration imparted by current compensation; region 5: at low values $\sigma \lesssim 10^{-7}$ (see text for details), micro-turbulence weakens the perpendicular current, the filamentation instability likely dominates the physics; in region 4 , microturbulence does weaken the perpendicular current, but the filamentation mode could not grow (above the diagonal line $\gamma_{\mathrm{sh}}^{2} \sigma \xi_{\mathrm{b}}^{-1}=1$ ) without the deceleration imparted by current compensation. See the text for further details.

The domain of influence of this instability in a phase diagram of relativistic collisionless shocks is illustrated in Fig. 5. At high magnetization, the shock is known to be mediated by magnetic reflection and the synchrotron maser instability, e.g. 25 28. At very low magnetizations, the Weibel/filamentation mode can structure the shock, as demonstrated by PIC simulations in the unmagnetized limit, e.g. [13. However, this same filamentation instability cannot grow above the diagonal line corresponding to $\gamma_{\mathrm{sh}}^{2} \sigma \xi_{\mathrm{b}}^{-1} \gtrsim 1$, as discussed above, in the absence of deceleration imparted by current compensation. In contrast, the current-driven instability can grow for any value of the shock Lorentz factor, provided $\sigma \lesssim 10^{-2}$; this determines its zone of influence illustrated in Fig. 5 .

Of course, once the background plasma has been decelerated through current compensation, the criterion for the growth of the Weibel/filamentation instability is itself modified; repeating the above arguments in $\mathcal{R}$, one finds $\sigma \lesssim \xi_{\mathrm{b}}^{3}$, which remains more stringent than for the current-driven filamentation. The two instabilities can then operate in conjunction in the precursor of relativistic shocks for $\sigma \lesssim \xi_{\mathrm{b}}^{3}$, thanks to the deceleration imparted by current compensation; however, in this region, the Weibel/filamentation instability still grows more slowly than the current-driven instability in the $\mathcal{R}$ frame.
Which instability prevails in this zone should be determined by PIC simulations. We comment further on this issue in the following.

The micro-turbulence not only builds up the shock at low magnetization, it also provides the needed source of scattering for the relativistic Fermi process. The analysis of particle trajectories actually leads to the following sine qua non condition for a proper relativistic Fermi process, see Ref. 23]:

$$
\sigma \lesssim \epsilon_{B, \mathrm{~d}}^{2}
$$

where $\epsilon_{B, \mathrm{~d}} \sim 10^{-2}-10^{-1}$ represents the typical value of $\epsilon_{B}$ downstream of the shock, assuming a micro-turbulence on scales $c / \omega_{\mathrm{p}}$. In this regard, the current-driven instability also plays a key role in triggering relativistic Fermi acceleration: by building up the micro-turbulence for any value of the shock Lorentz factor, it gives rise to the Fermi process in a large fraction of parameter space, whereas the standard Weibel/filamentation instability would be limited to the region $\sigma \lesssim \gamma_{\mathrm{sh}}^{-2} \xi_{\mathrm{b}}$. In this respect, we note that the transverse kinetic energy flux is a factor $\left|u_{y} / u_{x, \infty \mid \mathcal{S}}\right| \simeq \xi_{\mathrm{b}}$ of the incoming kinetic energy flux along $\boldsymbol{x}$, which implies that the waves excited through the current-driven instability may potentially grow up to a fraction $\epsilon_{B} \sim \xi_{\mathrm{b}}$, enough to mediate the shock.

The picture that we are drawing here seems supported by recent PIC simulations. In particular, one expects to observe a precursor of scale height $\sim c / \omega_{\mathrm{c}}$ if the currentdriven instability develops, since $c / \omega_{c}$ sets the scale over which the perpendicular current is generated; in contrast, the scale height of the precursor in Weibel/filamentation mediated shocks should be independent of that scale. The simulations reported in 19 precisely find a precursor with a scale height $\simeq 2 c / \omega_{\mathrm{c}}$ (as evaluated from their figure 7$)$; these simulations use $\gamma_{\mathrm{sh}}=21$ and $10^{-4} \lesssim \sigma \lesssim 10^{-3}$, values for which the Weibel/filamentation alone could not be excited, contrary to the current-driven instability. Moreover, the magnetic field appears structured in sheets parallel to the $x-y$ plane in these simulations rather than in filaments oriented along $\boldsymbol{x}$ (see their fig. 5), as would be expected for a standard Weibel/filamentation instability. Although our present linear analysis cannot predict the topology of the structures grown from the currentdriven instability, we find that maximal growth occurs for $k_{y} \ll k_{z}$, assuming a vanishing $k_{x}$, suggesting that the $z$-dependence is an important factor; one should of course investigate these issues with dedicated PIC simulations for a proper comparison to the above simulations.

Furthermore, the simulations of Ref. [19] report no dependence on the shock Lorentz factor, whereas a clear signature should be seen for the Weibel/filamentation mode, when the line $\gamma_{\mathrm{sh}}^{2} \sigma \xi_{\mathrm{b}}^{-1}=1$ is crossed. In the present picture, this independence is understood to result from the deceleration to $\gamma_{\mathcal{R} \mid \text { sh }} \sim 1 / \xi_{\text {b }}$ imparted by current compensation, the precursor playing the role of a buffer.

As the magnetization decreases, the micro-turbulence in the shock precursor plays an increasingly important role 
in scattering the supra-thermal particles. If this microturbulent scattering comes to dominate the transport of supra-thermal particles, it will randomize the tangential components of their velocities, hence it will randomize the perpendicular current itself; conversely, if coherent gyration around the background field prevails, the suprathermal particle current can indeed be considered as rigid. This allows to derive a critical value of $\sigma$, below which the back-reaction of the micro-turbulence becomes important, and above which our assumption of a rigid external current is valid, as follows. During a time interval $t_{\mathcal{R}}$, the particle acquires a rms deflection $\delta \theta \sim\left(\nu_{\mathrm{s} \mid \mathcal{R}} t_{\mathcal{R}}\right)^{1 / 2}$ through microturbulent scattering; in order for the particle to gyrate back to the shock front, it needs to be deflected away from the shock normal by an angle $\delta \theta_{\mathcal{R}} \sim 1 / \gamma_{\mathcal{R} \mid \text { sh }} \sim \xi_{\text {b }}$ in the $\mathcal{R}$ frame, see e.g. [22,29,30]; therefore, micro-turbulent scattering dominates over coherent gyration if $\delta \theta \gtrsim \delta \theta_{\mathcal{R}}$ for $t_{\mathcal{R}} \sim \delta \theta_{\mathcal{R}} / \omega_{\mathrm{c} \mid \mathcal{R}}$, the latter corresponding to the time taken to achieve a deflection of $\delta \theta_{\mathcal{R}}$ through coherent gyration. Using the fact that supra-thermal particles have typical Lorentz factor $\gamma_{\mathrm{sh}} / \xi_{\mathrm{b}}$ in the $\mathcal{R}$ frame, assuming that the micro-turbulence peaks on skin depth scales in this frame, one finds that coherent gyration remains a good approximation if $\sigma \gtrsim \xi_{\mathrm{b}}^{2} \epsilon_{B, \mathrm{u}}^{2}, \epsilon_{B, \mathrm{u}}$ characterizing the strength of the micro-turbulence in the shock precursor. Therefore, for $\sigma \gtrsim 10^{-7}$, coherent gyration is expected to prevail even up to the large values of $\epsilon_{B, \mathrm{u}}$ reached close to the shock front, supporting our assumption of a rigid external current. At lower values of $\sigma$, one should still expect the current-driven instability to develop at the tip of the precursor, where $\epsilon_{B, \mathrm{u}}$ is small; how the whole precursor is structured in this limit remains open for further study.

This current-driven instability thus modifies the paradigm according to which weakly magnetized $(\sigma \ll 1)$ shock waves are mediated by the Weibel/filamentation mode. Actually, the only region in parameter space where one can expect the Weibel/filamentation mode to grow in the absence of current-driven filamentation is $\sigma \lesssim \gamma_{\mathrm{sh}}^{-2} \xi_{\mathrm{b}}$.

The existence of this current-driven instability opens new avenues of research. In the field of collisionless shock physics, one must notably explore the exact interplay between this instability and the Weibel/filamentation mode in the region in which they can both grow, using dedicated PIC simulations. One must also understand how this instability shapes the magnetic field structure of the precursor, since the properties of the turbulence directly impact the acceleration process, e.g. [22]. This instability should also play an important role at the termination shock of pulsar winds, for which one expects $\sigma \lesssim 10^{-2}$ and $\gamma_{\mathrm{sh}} \gg 100$; which role and whether it can explain the remarkable (and unexpected) acceleration efficiency of such shocks [31], remains to be understood.

\section{REFERENCES}

[1] Medvedev M. V. and Loeb A., ApJ, 526 (1999) 697.

[2] Sentoku Y., Mima K., Kaw P. and Nishikawa K., PRL, 90 (2003) 155001.

[3] Bret A., Firpo M.-C. and Deutsch C., Phys. Rev. E, 70 (2004) 046401.

[4] Wiersma J. and Achterberg A., Astron. Astrophys., 428 (2004) 365.

[5] Lyubarsky Y. and Eichler D., ApJ, 647 (2006) 1250.

[6] Bret A., Gremillet L., Bénisti D. and Lefebvre E., PRL, 100 (2008) 205008.

[7] Bret A., Gremillet L. and Bénisti D., Phys. Rev. E, 81 (2010) 036402.

[8] Lemoine M. and Pelletier G., MNRAS, 417 (2011) 1148.

[9] Shaisultanov R., Lyubarsky Y. and Eichler D., ApJ, 744 (2012) 182.

[10] Kato T. N. and Takabe H., $A p J L, 681$ (2008) L93.

[11] Spitkovsky A., ApJL, 673 (2008) L39.

[12] Nishikawa K.-I., Niemiec J., Hardee P. E., Medvedev M., Sol H., Mizuno Y., Zhang B., Pohl M., Oka M. and Hartmann D. H., ApJL, 698 (2009) L10.

[13] Spitkovsky A., ApJL, 682 (2008) L5.

[14] Martins S. F., Fonseca R. A., Silva L. O. and Mori W. B., ApJL, 695 (2009) L189.

[15] Sironi L. and Spitkovsky A., ApJ, 698 (2009) 1523.

[16] Keshet U., Katz B., Spitkovsky A. and Waxman E., ApJL, 693 (2009) L127.

[17] Haugbølle T., ApJL, 739 (2011) L42.

[18] Sironi L. and Spitkovsky A., ApJ, 726 (2011) 75.

[19] Sironi L., Spitkovsky A. and Arons J., ApJ, 771 (2013) 54.

[20] Lemoine M., Pelletier G. and Revenu B., ApJL, 645 (2006) L129.

[21] Gedalin M., Smolik E., Spitkovsky A. and Balikhin M., EPL (Europhysics Letters), 97 (2012) 35002.

[22] Plotnikov I., Pelletier G. and Lemoine M., MNRAS, 430 (2013) 1280.

[23] Lemoine M. and Pelletier G., MNRAS, 402 (2010) 321.

[24] Lemoine M., Pelletier G., Gremillet L. and PlotNIKOV I., MNRAS, 440 (2014) 1365.

[25] Alsop D. and Arons J., Phys. Fluids, 31 (1988) 839.

[26] Hoshino M. and Arons J., Physics of Fluids B, 3 (1991) 818.

[27] Hoshino M., Arons J., Gallant Y. A. and Langdon A. B., Astrophys. J., 390 (1992) 454.

[28] Gallant Y. A., Hoshino M., Langdon A. B., Arons J. and Max C. E., Astrophys. J., 391 (1992) 73.

[29] Achterberg A., Gallant Y. A., Kirk J. G. and Guthmann A. W., MNRAS, 328 (2001) 393.

[30] Milosavljević M. and Nakar E., Astrophys. J., 651 (2006) 979

[31] Arons J., Sp. Sc. Rev., 173 (2012) 341. 